\title{
Treatment Model in Children with Speech Disorders and Its Therapeutic Efficiency
}

\author{
Luciana Barberena ${ }^{1}$ Márcia Keske-Soares ${ }^{1}$ Taís Cervi $^{1}$ Mariane Brandão ${ }^{1}$ \\ 1 Speech Therapy Department, Universidade Federal de Santa Maria, \\ Santa Maria, RS, Brazil \\ Int Arch Otorhinolaryngol 2014;18:283-288. \\ Address for correspondence Luciana Barberena, MSc, Department of \\ Fonoaudiologia, Universidade Federal de Santa Maria, Rua Tuiuti, 520, \\ apto 202, Santa Maria, Rio Grande do Sul 97015-660, Brazil \\ (e-mail: lucianabarberena@hotmail.com).
}

\begin{abstract}
Keywords

- speech

- articulation disorders

- speech language and hearing sciences

- speech therapy

- generalization

Introduction Speech articulation disorders affect the intelligibility of speech. Studies on therapeutic models show the effectiveness of the communication treatment.

Objective To analyze the progress achieved by treatment with the ABAB-Withdrawal and Multiple Probes Model in children with different degrees of phonological disorders. Methods The diagnosis of speech articulation disorder was determined by speech and hearing evaluation and complementary tests. The subjects of this research were eight children, with the average age of 5:5. The children were distributed into four groups according to the degrees of the phonological disorders, based on the percentage of correct consonants, as follows: severe, moderate to severe, mild to moderate, and mild. The phonological treatment applied was the ABAB-Withdrawal and Multiple Probes Model. The development of the therapy by generalization was observed through the comparison between the two analyses: contrastive and distinctive features at the moment of evaluation and reevaluation.

Results The following types of generalization were found: to the items not used in the treatment (other words), to another position in the word, within a sound class, to other classes of sounds, and to another syllable structure.

Conclusion The different types of generalization studied showed the expansion of production and proper use of therapy-trained targets in other contexts or untrained environments. Therefore, the analysis of the generalizations proved to be an important criterion to measure the therapeutic efficacy.
\end{abstract}

\section{Introduction}

For most children, between birth and the age of 5 , the maturation of phonological knowledge occurs in a gradual process, in a nonlinear manner and with individual variations, resulting in the establishment of a system consistent with the adult target. ${ }^{1}$ However, in some children, phonological systems develop that differ from the language in their environment; this is called phonological/speech disorder.

Most children with communication disorders have at least some difficulty in the phonological level of the language, in their knowledge of phonetic segments and phonological rules. ${ }^{2}$ This

received

February 6, 2014

accepted

April 4, 2014

published online

May 28, 2014
DOI http://dx.doi.org/

10.1055/s-0034-1376885. ISSN 1809-9777. difficulty affects speech intelligibility, in some cases making understanding of their language impossible. The phonological disorders can be classified according to the degree of severity. This classification can help in clinical practice, contributing to the use of different therapeutic procedures and therapeutic models with a phonological basis. Studies with therapeutic models and the possibility to relate different models according to the degree of severity of phonological disorder are relevant and provide important advances in speech therapy.

Phonological therapy aims at improving the child's speech and facilitating the reorganization of his or her phonological system, thereby increasing the effectiveness of communication. ${ }^{3}$
Copyright $\odot 2014$ by Thieme Publicações License terms Ltda, Rio de Janeiro, Brazil

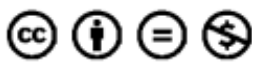


Thus, the treatment must prioritize the generalization. The generalization is characterized by the expansion of production and proper use of target language practiced in therapy in other contexts or environments that were not focused on in the speech therapy. ${ }^{4}$ The generalization can also be defined as expansion or transfer of learning, and it is the most important criterion to measure the therapeutic efficacy. ${ }^{5}$

The generalization occurs in different ways and can be seen when the child transfers use of the pattern learned to other words that were not targeted in therapy, when the child learns a sound in a particular position in the word and performs it correctly in other positions, when the child extends this learning to other sounds belonging to the same class of sound that has been learned or to different classes of what was learned, or when the child uses the phonological patterns that were learned in therapy to other patterns learned in external environments. ${ }^{4}$

The "ABAB-Withdrawal and Multiple Probes" is a model with phonological base, widely used in Brazil. ${ }^{6}$ With this model, it is possible to obtain various types of generalization during the speech therapy.

The objective of this study is to analyze the different types of structural generalization in children with phonological disorders, obtained by the ABAB-Withdrawal and Multiple Probes Model, namely: to items that were not used during the treatment (to other words); to another position in the word; within a class of sounds; to another class of sounds; and to other syllabic structure.

\section{Methods}

Eight children with phonological disorders participated in the therapy, four boys and four girls, with an average age of 5:5 years old at the beginning of the treatment. All the children received their phonological treatment in the Speech Therapy Service of the institution where this work was performed.

This research is part of a master's thesis whose project was properly submitted to and approved by the Committee of Ethics and Research of the institution, registered under number 071/03. Prior parental consent was obtained along with signed informed consent forms.

The children were included if they did not present changes in their otorhinolaryngology, neurological, and audiological evaluations, conducted in the same department. The children's speech was also evaluated by assessment of receptive and expressive language, the oral sensorimotor system, psychomotricity, auditory discrimination, and phonological assessment, which consisted of collecting and analyzing the speech data. The phonological assessment was applied based on the Phonological Evaluation of Children (PEC) and revealed disorders at the phonological level and reduced phonetic inventory, with impaired speech intelligibility. ${ }^{2}$ This instrument also enabled, by means of contrastive analysis, classification according to the degree of severity of phonological disorders, from the percentage of correct consonants. ${ }^{7}$ From the result of the percentage of correct consonants, the phonological disorder was classified according to the degree of severity, and the children were divided into four groups: mild
(86 to $00 \%$ ), mild to moderate (66 to $85 \%$ ), moderate to severe (51 to $65 \%$ ), and severe deviation ( $<50 \%$ ).

The ABAB-Withdrawal and Multiple Probes Model was applied as phonological treatment, ${ }^{6}$ considering the Implicational Model of Complexity of Features in the choice of target sounds used in each treatment cycle. ${ }^{8}$ The ABAB-Withdrawal and Multiple Probes initially involves the collection of data from each child (A1). In this collection, the children's spontaneous speech was recorded and analyzed and the PEC instrument was applied. Afterward, the altered distinctive features were identified and the target sound was determined for the treatment of each individual ( $\mathbf{- T a b l e ~} \mathbf{1}$ ).

The therapeutic intervention starts in the first treatment cycle (B1) and lasts for 5 weeks (nine sessions), with two 45-minute therapy sessions a week. Basic Target Tests (BTT) are also performed during the treatment cycles and used to evaluate the child's performance through the cycles.

\section{Table 1}

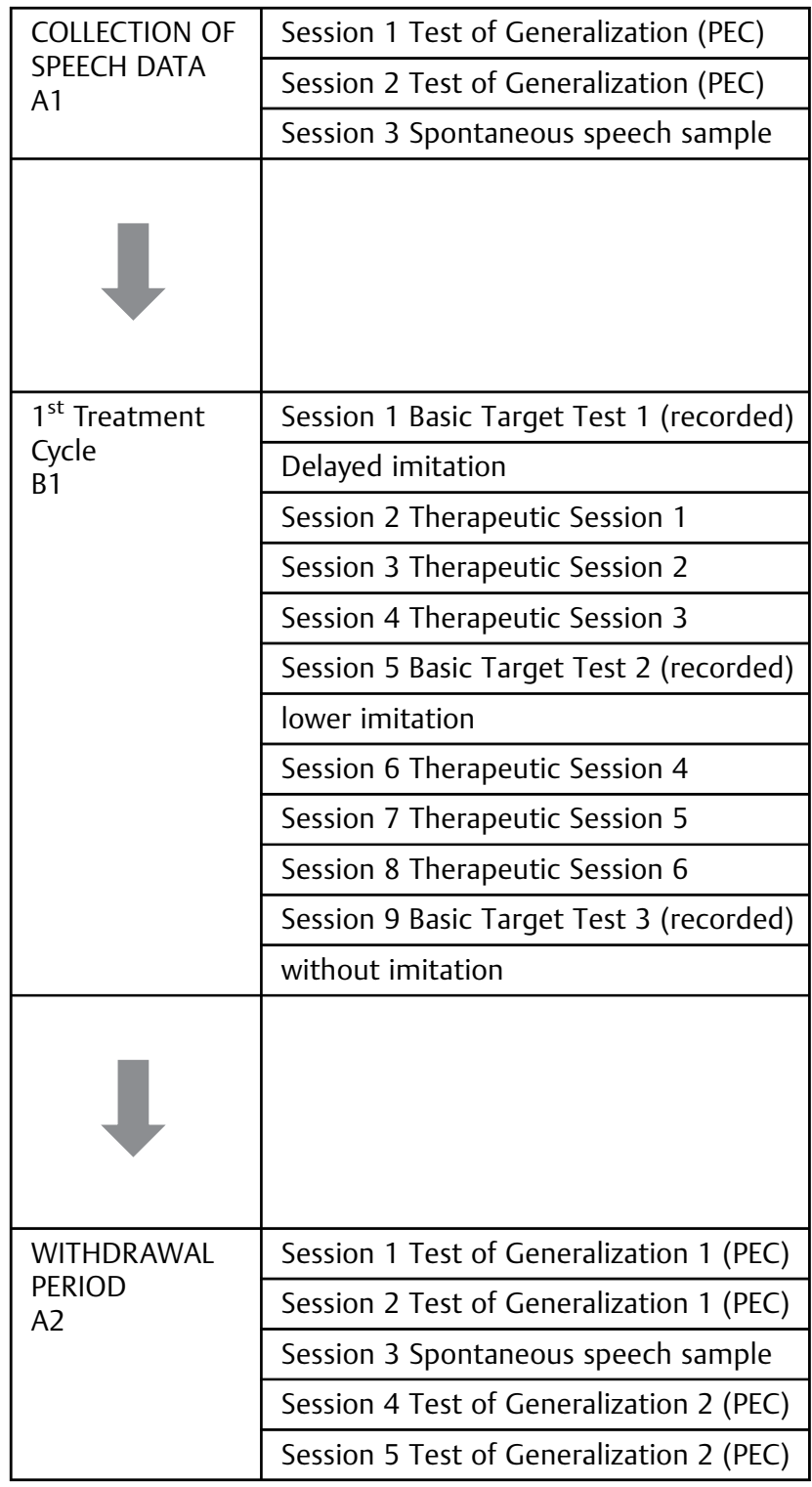

Abbreviations: A1, collection of data from each child; B1, first treatment cycle; A2, period of withdrawal. 
After the first intervention, there is a period of withdrawal (A2); in this period, the planned tests are administered without direct intervention upon the sounds chosen as targets. During this approximately 3-week period, which corresponds to five sessions, the tests of generalization are given and spontaneous speech samples are collected from the child.

The treatment follows according to the necessity of the case, through another cycle of treatment (B2) and other withdrawal periods.

The treatment model used in this research follows, considering the tests included for each cycle (BTT) and the tests of generalization in the periods of withdrawal.

The analysis of the therapy evolution (generalization) was observed through comparisons between contrastive and distinctive features analysis, as well as from the evaluations and reevaluations in the tests of generalization and basic target tests performed in the therapy model.

From the treatment, it was possible to analyze the occurrence of the following types of generalizations in the different groups of children according to the degree of severity:

1. To items not used in the treatment. This type of generalization occurs when the child is able to produce the practiced sounds not only in the words used during therapy but also in other words that were not practiced.

2. To another position in the word. This type of generalization occurs when the correct production of the target sound occurs not only in the stimulated position but also in the other positions in the word.

3. Within a class of sounds. This type of generalization occurs when the child transfers the learning to other sounds belonging to the same class of the sound practiced, without any direct intervention.

4. To other classes of sounds. Such generalization occurs when there is an extension of the learning to sounds belonging to different classes of the stimulated sound.

5. To another syllable structure. This type of generalization occurs when the child acquires the ability to produce a practiced sound on a more complex syllable structure than the one in the therapy.

Fisher exact test was used to compare the findings of the phonological system before and after the treatment by the ABAB-Withdrawal and Multiple Probes Model among different degrees of severity of phonological disorder.

\section{Results}

- Fig. 1 shows the average percentage of generalizations to items not used in the treatment (other words besides those used in therapy). This type of generalization has the largest number of occurrences for the mild to moderate group (MMG; 90.9\%), followed by the mild group (MG; 78.1\%), severe group (SG; 73.1\%), and moderate to severe group (MSG; 64\%). This kind of generalization was observed in other studies. ${ }^{9-13}$ In this study, all groups showed this kind of generalization, but the MMG showed better results compared with the other groups.

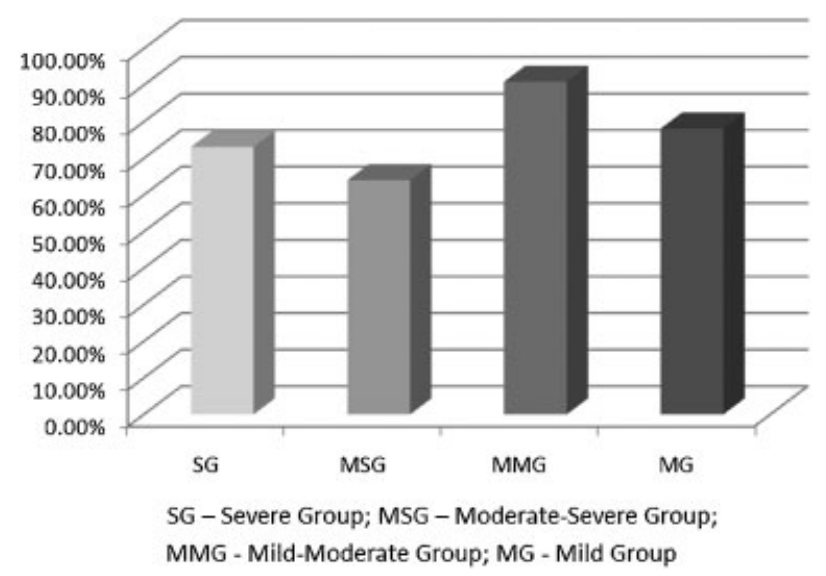

Fig. 1 Average percentage of generalizations for items not used in the treatment (other words besides those used in therapy). Abbreviations: MG, mild group; MMG, mild to moderate group; MSG, moderate to severe group; SG, severe group.

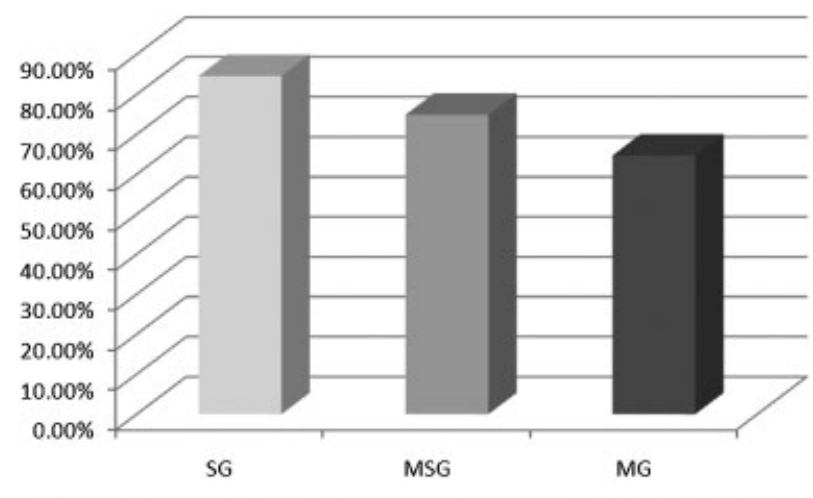

SG - Severe Group; MSG - Moderate-Severe Group; MG - Mild Group

Fig. 2 Average percentage of generalizations to another position in the word. Abbreviations: MG, mild group; MMG, mild to moderate group; MSG, moderate to severe group; SG, severe group.

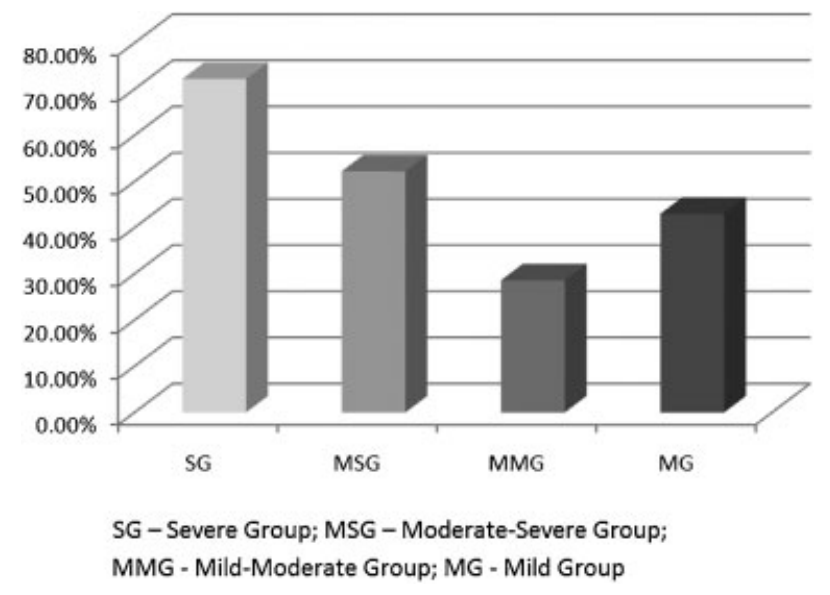

Fig. 3 Average percentage of generalizations within a class of sounds. Abbreviations: MG, mild group; MMG, mild to moderate group; MSG, moderate to severe group; SG, severe group. 


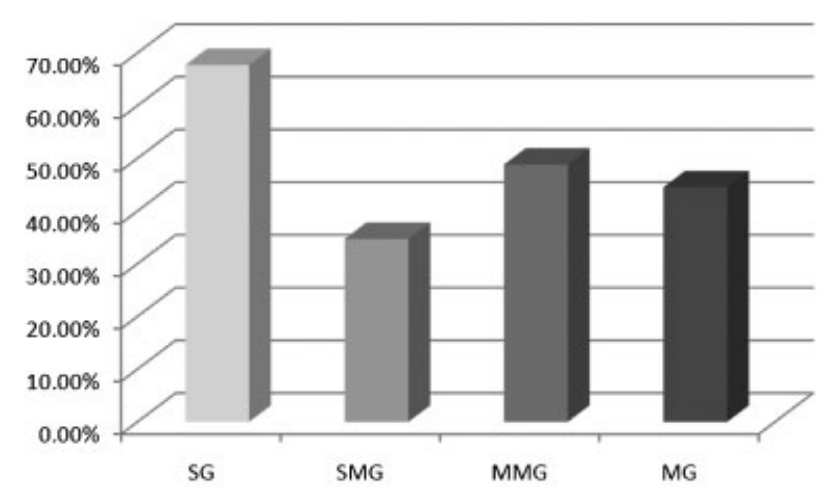

SG - Severe Group; MSG - Moderate-Severe Group; MMG - Mild-Moderate Group; MG - Mild Group

Fig. 4 Average percentage of generalizations to other classes of sounds. Abbreviations: MG, mild group; MMG, mild to moderate group; MSG, moderate to severe group; SG, severe group.

-Fig. 2 shows the average percentage of generalizations to another position in the word. This type of generalization occurred in the highest number of sounds in the SG (84.6\%) and MSG (74.9\%) and finally the MG (64.7\%). The MMG did not exhibit this kind of generalization due to the choice of the selected target sounds occurring only in positions in which they were treated.

-Fig. 3 shows the average percentages of generalizations within a class of sounds. This type of generalization also had most occurrences in SG (72.2\%), followed by MSG (52.2\%), MG (43\%), and MMG (28.6\%). The MMG benefited least from this kind of generalization. This fact is probably justified by the target sound chosen in the sound class stimulated in this group, which seems not to have benefited the generalization in the same sound class.

-Fig. 4 shows the average percentage of generalizations to other classes of sounds. This type of generalization also occurred to a greater number of sounds in the SG (67.7\%), followed by the MMG (48.8\%), MG (44.5\%), and MSG (34.7\%). Although all groups showed changes in the proposed treatment, the MSG benefited the least from this kind of generalization. The results are also found in other studies. 5,14

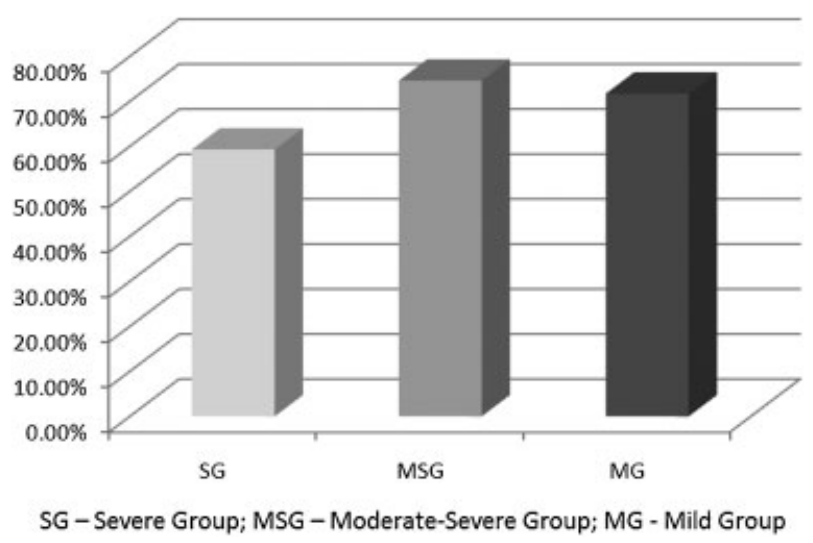

Fig. 5 Average percentage of generalizations to other syllabic structure. Abbreviations: MG, mild group; MMG, mild to moderate group; MSG, moderate to severe group; SG, severe group.
Table 2 Phonological system pre- and posttreatment of the eight studied children according to the degree of severity of phonological disorder

\begin{tabular}{|l|l|}
\hline Averages of groups & $\mathrm{p}$ value \\
\hline Severe and moderate to severe & 0.016 \\
\hline Severe and mild to moderate & 0.0354 \\
\hline Severe and mild & 0.0049 \\
\hline $\begin{array}{l}\text { Moderate to severe and mild } \\
\text { to moderate }\end{array}$ & 0.8379 \\
\hline Moderate to severe and mild & 0.8479 \\
\hline Mild to moderate and mild & 0.5846 \\
\hline
\end{tabular}

Fisher exact test $(p \leq 0.05)$.

-Fig. 5 shows the average percentage of generalizations to other syllabic structures. This type of generalization occurred to a greater number of sounds in the MSG, followed by the MG and SG. It was not possible to observe this type of generalization in the MMG because it is not possible to perform this sound in another syllable structure besides the one used in the treatment. Generalizations to other syllabic structure were found in other studies. ${ }^{11,15}$

When the initial and final phonological systems of all groups were compared, children from the SG were those who had a greater number of sounds in comparison to the other groups (-Table 2). Possibly the deviations of severe degree had higher evolution due to the establishment of a greater number of distinctive features. The greater the severity of the phonological disorder, the more phonemes are established in the phonological system of the child. ${ }^{16}$

\section{Discussion}

The generalization to items not used in the treatment (other words besides those used in therapy; - Fig. 1) showed that the children in this study were able to use the pattern learned in other words that were not practiced in the therapy session. Thus, the children produced the target sounds not only in the words they were trained, but also in other words not analyzed. This indicates that few words are needed to teach a particular sound because its use can be transferred to many others.

In one study, ${ }^{17}$ the therapy performed with the ABABWithdrawal and Multiple Probes was compared with two other models with phonological basis and the authors noticed generalizations to items not used in the treatment. The ABAB-Withdrawal and Multiple Probes Model was effective in all degrees of severity of phonological disorder and had a higher percentage of this type of generalization compared with other models.Generalizations to another position in the word (-Fig. 2) indicated that when learning a phoneme in a given word position, the child did it in other positions not worked in therapy. This fact highlights that the use of the sound was not just restricted to the position where it was worked with, and it shows that, when it is possible, the children in this study were able to transfer the sound into different positions from those that were learned in 
therapy sessions. The generalization to another position in the word was found in other studies. ${ }^{9-13,15,17}$

Although - Fig. 3 shows the average percentages of generalizations within a class of sounds, there are significant generalizations within a class of sounds in most groups. This type of generalization showed that the children in this study, when learning a phoneme, extended this learning to other phonemes belonging to the same class of sound that was practiced. This fact indicated that the sounds that relate to each other can be acquired without a direct intervention in all of them. In the treatment of only one incorrect sound or only one aspect of a standard error, various other incorrect sounds can be corrected, providing, as a result, a more efficient treatment. The generalization within a class of sounds was also found in other studies. ${ }^{10-13}$ The generalization to other classes of sounds (-Fig. 4) is a more complex type of generalization. When learning a sound, the child extends this learning to other phonemes that do not belong to the same class of target sound. This type of generalization is quite interesting and effective, as the knowledge of other classes of sounds in these children made it possible for a reduction in the therapy period because other whole classes of sounds have been learned without direct intervention.

In another study, ${ }^{18}$ the therapy performed with the $A B A B$ -Withdrawal and Multiple Probes was compared with two other models of phonological basis and the authors also noticed generalizations within a sound class and another class of sounds. ${ }^{18}$ This research found that the ABAB-Withdrawal and Multiple Probes was effective in all degrees of severity of phonological disorders.The generalization to other syllabic structure (-Fig. 5) is important as it allows that sounds worked in complex syllabic structures are extended to simpler structures, such as in the case of consonant clusters, facilitating the learning process. In some cases, it is also possible for the child to generalize sounds from simpler structures into more complex structures.

-Fig. 5 shows a low percentage of generalizations to other syllabic structure in the SG when compared with the other types of generalization in this group. It must be highlighted that, in choosing the target sounds for the therapy, occurrence for most of these sounds in other syllable structures was not possible, which explains the low percentage in this group for this type of generalization.

It is important to mention that, although the subjects were classified according to the degree of severity of phonological disorder, individual variations during the acquisition process should also be considered. The phonological acquisition is nonlinear, and the data analysis indicates that during the therapeutic process attention should be focused on the generalization of practiced phonemes. ${ }^{19}$

One study assessed the progress obtained by the model of phonological therapy ABAB-Withdrawal and Multiple Probes. This model was compared with two other ones also with a phonological basis. The statistical analysis showed that the number of phonemes established in the phonological system differed from the initial and final evaluations in the three models. ${ }^{3}$ These results corroborate the findings of another study in which this model of therapy was also compared with two other models and showed important generalizations in the phonological systems of children. ${ }^{15}$

One study also demonstrated that the application of $A B A B$ -Withdrawal and Multiple Probes revealed favorable developments regarding the acquisition of the phonological system, regardless of the severity of phonological disorder and age. ${ }^{16}$ However, the larger the number of sessions, the more sounds were acquired.

In this research, the proposed treatment proved to be effective for all degrees of severity of phonological disorder, but the severe degree of deviation revealed a higher percentage in three of the five types of generalization analyzed. It is important to note that other groups also benefited, even MG and MMG, which in some types of generalizations showed lower evolution when compared with the other groups. This fact can be explained in the study in which a model of therapy with phonological basis was applied. ${ }^{13}$ In this study, it was noted that the group of subjects with mild phonological disorder showed lower phonological changes and fewer generalizations when compared with other groups, because this group presents phonological systems with a few alterations, therefore with less possibilities of generalization.

\section{Conclusion}

The different types of generalization studied showed the expansion of production and proper use of therapy-trained targets in other contexts or untrained environments. Therefore, the analysis of the generalizations proved to be an important criterion to measure therapeutic efficacy. Using this criterion, it is possible to state that the treatment proposed by the ABAB-Withdrawal and Multiple Probes Model provided evident improvement in the speech of children with speech disorders.

All the groups representing the degrees of severity of phonological disorder benefited from the treatment. Most of the observed generalizations occurred in the SG, as well as the establishment of a greater number of sounds in the phonological system after the treatment when compared with the other groups.

\section{References}

1 Lamprecht RR. (Org.). Phonological Acquisition of Portuguese: Development Profile and Allowances for Therapy. 1a ed. Porto Alegre. Art Med 2004

2 Yavas MS, Hernandorena CLM, Lamprecht RR. Phonological Assessment of Child. 2a ed. Porto Alegre. Art Med 2003

3 Mota HB, Keske-Soares M, Bagetti T, Ceron MI, Melo Filha MGC. Comparative analyzes of the efficiency of three different models of phonological therapy. Pro Fono 2007;19(1):67-74

4 Elbert M, Gierut JA. Handbook of Clinical Phonology. 1st ed. London, UK: Taylor and Francis; 1986

5 Gierut JA. Complexity in phonological treatment: clinical factors. Lang Speech Hear Serv Sch 2001;32(1):229-241

6 Tyler AA, Figurski GR. Phonetic inventory changes after treating distinctions along an implicational hierarchy. Clin Linguist Phon 1994;8(2):91-107

7 Shriberg LD, Kwiatkowski J. Phonological disorders I: a diagnostic classification system. J Speech Hear Disord 1982;47(3):226-241 
8 Mota HB. Segmental acquisition of Portuguese: an implicational model of feature complexity. Letras Hoje 1997;32(4):23-47

9 Powell TW, Elbert M. Generalization following the remediation of early- and later-developing consonant clusters. J Speech Hear Disord 1984;49(2):211-218

10 Mota HB, Pereira LF. The generalization in phonological therapy in speech disorders: experience with two children. Pro Fono 2001; 13(2):141-146

11 Blanco APF. The generalization in the model modified cycles in patients with different degrees of severity of phonological disorder [dissertation]. Santa Maria, Brazil: Universidade Federal de Santa Maria, Santa Maria, Center of Health Sciences; 2003

12 Mota HB, Bagetti T, Keske-Soares M, Pereira LF. The generalization in subjects with mild-moderate phonological disorder treated by the model of maximum oppositions. Soc Bras Fonoaudiol 2004;9(2):102-111

13 Bagetti T, Mota HB, Keske-Soares M. Model modified maximum opposition: a proposed treatment for phonological disorders. Soc Bras Fonoaudiol 2005;10(1):36-14.
14 Keske-Soares M. Speech therapy based on implicational hierarchy of distinctive features applied in children with phonological disorders [thesis]. Porto Alegre, Brazil: Pontifical Catholic University of Rio Grande do Sul, Porto Alegre, Faculty of Letters; 2001

15 Mota HB, Keske-Soares M, Ferla A Zasso LV, Dutra LV. Comparative study of generalization in three model of therapy for phonological disorders. Saúde: Rev Centro Ciência Saúde 2002;28(1):36-47

16 Ceron MI, Pagliarin KC, Keske-Soares M. Advances in the treatment of children with phonological disorders. Int Arch Otorhinolaryngol 2013;17(2):189-195

17 Ceron MI, Keske-Soares M. Phonological therapy: the generalization to items not used in the treatment (other words). Rev CEFAC 2007;9(4):453-460

18 Ceron MI, Keske-Soares M. Phonological therapy: the generalization inside a sound class and for other sound classes. Rev CEFAC 2008;10(3):311-320

19 Keske-Soares M, Pagliarin KC, Ghisleni MRL, Lamprecht RR. Non linear acquisition during the therapeutic process. Letras de Hoje 2008;43(3):22-26 